\title{
Editorial
}

\section{Caspases and their many biological functions}

\author{
S Kumar*1,2 \\ Cell Death and Differentiation (2007) 14, 1-2. doi:10.1038/sj.cdd.4402064; published online 3 November 2006
}

By the time this editorial goes to press, it will be almost 15 years since the discovery in 1992 of the first caspase, interleukin-1 $\beta$-converting enzyme (ICE), which is now known as caspase-1. ${ }^{1,2}$ As the name suggests ICE/caspase- 1 was discovered as an enzyme that proteolytically processes interleukin- $1 \beta$ precursor to generate active interleukin- $1 \beta .^{1,2}$ The discovery and initial characterisation of ICE provided no clues to the role of ICE-like proteases in cell death. It was only the cloning of the Caenorhabditis elegans cell death gene ced3 in 1993 which generated evidence that CED-3/ICE-like proteases, now widely known as caspases (cysteinyl aspartate proteinases), play key roles in apoptosis execution. ${ }^{3}$ Besides ICE, one of the first known mammalian homologues of CED-3 was Nedd2 (now known as caspase-2), initially cloned in 1992 as a developmentally regulated mouse gene. $^{4,5}$ Ironically, despite being the closest mammalian homologue of CED-3, the function of caspase-2 still remains an enigma. Nevertheless, the discovery of CED-3 heralded a flurry of publications in subsequent years on cloning and characterisation of a large number of caspases from various metazoans. It is fair to say that the impact of the discovery of caspases on the field of cell death has been enormous. At the time of writing this editorial a Pubmed (National Center of Biotechnology Information) search indicated around 26000 publications contain the word caspase in their title or abstract - which is remarkable given the short time since the discovery of the first caspase.

Despite the fact that the function of caspase- 1 is primarily to activate proinflammatory cytokines, much of the earlier work on caspases focused on their functions in apoptosis. Cell Death and Differentiation, then a nascent journal trying to find its way in a rapidly evolving and expanding field, published its first review on caspases in $1996 .{ }^{6}$ This followed in 1999 the publication of a comprehensive set of reviews covering all aspects of caspases. ${ }^{7-14}$ These reviews, contributed by many of leaders in caspase research, were highly successful and provided an excellent resource for researchers in the field for many years. A number of other stand-alone reviews on different aspects of caspase biology and function have been published by this journal since 1999. Given that a lot of new information has become available in the past 7 years since the publication of the 1999 caspase review series, the editors considered this an opportune time to publish an up-to-date compendium of reviews on caspases. As a result this issue of Cell Death and Differentiation carries seven focused reviews on various aspects of caspases. We also plan to publish additional reviews later this year on aspects of caspases that are not covered here.

Given that the first discovered function of caspases was in inflammation, ${ }^{1,2}$ inflammatory caspases are the subject of the first two, slightly overlapping but comprehensive, reviews by Martinon and Tschopp ${ }^{15}$ and Scott and Saleh. ${ }^{16}$ The proinflammatory caspases are only found in vertebrates, with caspase- 1 being the prototypic member of the family that also includes caspases-4, $-5,-11$ and $-12 .^{15,16}$ The article by Martinon and Tschopp ${ }^{15}$ focuses on these caspases and how their activation may be regulated by large multiprotein complexes, often termed inflammasomes. They describe the complexity of these caspase activation platforms as evident from multiple caspase-1 activating inflammasomes. They then discuss the signals that activate inflammasomes, the roles of inflammasomes in autoinflammatory disorders, innate and adaptive immune responses, and the regulation of inflammatory caspase activation. The review article by Scott and Saleh ${ }^{16}$ expands on the topic of inflammatory caspases, with particular emphasis on caspase-12 and its role in sepsis. The authors also discuss inflammatory caspase gene organisation, expression, regulation, substrates, and role in innate immunity and inflammatory disorders. In the last section of their article they summarise the potential role of inflammatory caspases in apoptosis in specific paradigms.

Genetic studies in $C$. elegans suggest that one of the conserved functions of caspases is to execute cell death. ${ }^{7}$ Consequently, the loss of ced-3 function leads to a block of all programmed death of somatic cells in the worm. In an article on apoptotic caspases I have summarised the in vivo and genetic evidence for the roles of individual caspases in programmed cell death. ${ }^{17}$ I have included studies with $C$. elegans and Drosophila caspases, along with the analyses of mammalian caspase gene knockouts in mice and have attempted to discuss the current understanding, controversies and unresolved issues relating to the role of caspases in apoptosis. The article also provides a brief description of the regulation of apoptotic caspases.

Recently it has become evident that some caspases, in addition to their apoptotic and/or proinflammatory functions,

\footnotetext{
${ }^{1}$ Department of Haematology, Hanson Institute, Institute of Medical and Veterinary Science, PO Box 14, Rundle Mall, Adelaide, South Australia 5000, Australia and ${ }^{2}$ The Department of Medicine, University of Adelaide, Adelaide, South Australia 5000, Australia

${ }^{*}$ Corresponding author: S Kumar, Department of Haematology, Hanson Institute/IMVS, PO Box 14, Rundle Mall, Adelaide, South Australia 5000, Australia.

E-mail: sharad.kumar@imvs.sa.gov.au
} 
also participate in cell proliferation, differentiation and survival. For example DRONC, the key cell death caspase in Drosophila, has been reported to have additional functions in compensatory proliferation, border cell migration, neural differentiation and spermatid individualisation, and caspase-8 in mammals is required for proliferation of $B, T$ and NK cells, and for the differentiation of monocytes. ${ }^{18}$ Several studies now suggest that the limited activation of some caspases often leads to a nonapoptotic outcome whereas global activation of the same caspases results in apoptosis. These topics and other caspases with nonapoptotic functions are discussed in an article by Lamkanfi et al. ${ }^{18}$ The authors also discuss how some caspases can induce $\mathrm{NF}_{\kappa} \mathrm{B}$ activation with or without exerting their proteolytic function. They then provide a compilation of 'non-apoptotic' caspase substrates and discuss how the cleavage of these substrates relates to the specific nonapoptotic functions of the given caspases.

Initiator caspase activation is mediated by the large adaptor-caspase complexes, often referred to as the apoptosomes. ${ }^{7,10}$ In $C$. elegans CED-3 is activated by a CED-4 complex, in Drosophila DRONC activation occurs via recruitment to an ARK complex, whereas activation of mammalian caspase- 8 and -9 is mediated by the death-inducing signaling complex (DISC) and the Apaf- 1 apoptosome, respectively. ${ }^{19}$ The article by Bao and Shi ${ }^{19}$ discusses these and the putative caspase-2 activation complex (termed PIDDosome) in light of extensive structural data that have recently become available. Authors also discuss various models of initiator caspase activation by the caspase activation complexes.

The activated caspases target various proteins in order to carry out their apoptotic or nonapoptotic functions. In addition to autocleaving to self-activate in activation complexes (inflammasomes, apoptosomes and PIDDosome), the main targets of initiator caspases are the effector (downstream) caspase such as caspases-3, -6 and $-7 .{ }^{7}$ Upon activation these caspases mediate the cleavage of a large number of cellular substrates. Timmer and Salvesen ${ }^{20}$ in their article on caspase substrates point out that of the hundreds of published putative targets of caspases only a few have been rigorously established as bona fide substrates in vivo. They discuss that many of the proteins cleaved in apoptotic cells probably represent innocent bystanders. They provide a detailed view of substrate specificity of caspases, the role of cleavage site conformation, caspase cleavage site prediction in putative substrates and discuss the consequences of caspasemediated cleavage of key substrate proteins.

Many natural inhibitors of caspases are known, including several viral and cellular proteins that either act as direct inhibitors of caspases or block the activation of caspases. ${ }^{13}$ Use of these inhibitors has played a major role in understanding caspase biology, enzymology and function, in addition to providing opportunity for pharmacological targeting of caspases in pathological conditions. ${ }^{7,13}$ Callus and Vaux focus on natural and synthetic inhibitors of caspases. ${ }^{21}$ In their article the authors discuss various modes of caspase inhibition by viral, cellular and chemical inhibitors. They also provide a compilation of data on the efficacy of inhibition by various caspase inhibitors and discuss the potential use of recently discovered small molecule caspase inhibitors as pharmacological agents.

Overall these review summarise a vast amount of information relating to caspase biochemistry, activation, regulation and function. I hope the readers of Cell Death and Differentiation will find these reviews as an up-to-date resource for information on caspase biology and function, useful both for research and teaching purposes.

1. Thornberry NA et al. Nature $1992 ; 356: 768-774$

2. Cerretti DP et al. Science 1992; 256: 97-100.

3. Yuan J et al. Cell 1993; 75: 641-652.

4. Kumar S, Tomooka Y, Noda M. Biochem Biophys Res Commun 1992; 185: 1155-1161.

5. Kumar S et al. Genes Dev 1994; 8: 1613-1626.

6. Kumar S, Lavin MF. Cell Death Differ 1996; 3: 255-267.

7. Nicholson DW. Cell Death Differ 1999; 6: 1028-1042.

8. Zheng TS et al. Cell Death Differ 1999; 6: 1043-1053.

9. Stennicke HR, Salvesen GS. Cell Death Differ 1999; 6: 1054-1059.

10. Kumar S. Cell Death Differ 1999; 6: 1060-1066.

11. Slee EA, Adrian C, Martin SJ. Cell Death Differ 1999; 6: 1067-1074.

12. Zeuner A et al. Cell Death Differ 1999; 6: 1075-1080.

13. Ekert PG, Silke J, Vaux DL. Cell Death Differ 1999; 6: 1081-1086.

14. Cecconi F. Cell Death Differ 1999; 6: 1087-1098.

15. Martinon F, Tschopp J. Cell Death Differ 2007; 14: 10-22.

16. Scott AM, Saleh M. Cell Death Differ 2007; 14: 23-31.

17. Kumar S. Cell Death Differ 2007; 14: 32-43.

18. Lamkanfi M et al. Cell Death Differ 2007; 14: 44-55.

19. Bao Q, Shi Y. Cell Death Differ 2007; 14: 56-65.

20. Timmer JC, Salvesen GS. Cell Death Differ 2007; 14: 66-72.

21. Callus B, Vaux DL. Cell Death Differ 2007; 14: 73-78. 